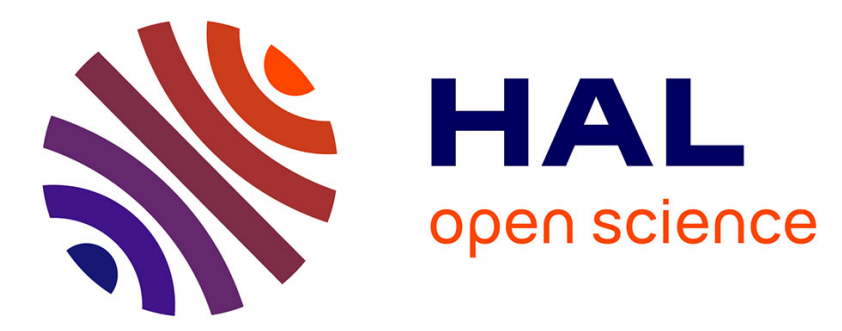

\title{
Theoretical analysis of a resonant quartz-enhanced photoacoustic spectroscopy sensor
}

Guillaume Aoust, Raphaël Levy, Myriam Raybaut, Antoine Godard, Jean-Michel Melkonian, Michel Lefebvre

\section{To cite this version:}

Guillaume Aoust, Raphaël Levy, Myriam Raybaut, Antoine Godard, Jean-Michel Melkonian, et al.. Theoretical analysis of a resonant quartz-enhanced photoacoustic spectroscopy sensor. Applied Physics B - Laser and Optics, 2017, 123 (2), pp.63. 10.1007/s00340-017-6640-z . hal-01499245

\section{HAL Id: hal-01499245 \\ https://hal.science/hal-01499245}

Submitted on 22 Nov 2021

HAL is a multi-disciplinary open access archive for the deposit and dissemination of scientific research documents, whether they are published or not. The documents may come from teaching and research institutions in France or abroad, or from public or private research centers.
L'archive ouverte pluridisciplinaire HAL, est destinée au dépôt et à la diffusion de documents scientifiques de niveau recherche, publiés ou non, émanant des établissements d'enseignement et de recherche français ou étrangers, des laboratoires publics ou privés.

\section{(ㅇ)(1) $\$$}

Distributed under a Creative Commons Attribution - NonCommerciall 4.0 International 


\title{
Theoretical analysis of a resonant quartz-enhanced photoacoustic spectroscopy sensor
}

\author{
Guillaume Aoust $^{1,2}$ (1) Raphael Levy $^{1} \cdot$ Myriam Raybaut $^{1} \cdot$ Antoine Godard $^{1}$. \\ Jean-Michel Melkonian ${ }^{1} \cdot$ Michel Lefebvre $^{1}$
}

\begin{abstract}
In this paper, we report the first analytical model for quartz-enhanced photoacoustic spectroscopy in combination with an acoustic resonator. A generalized fundamental equation is proposed to model the photoacoustic effect, taking into account the coupling between the tuning fork and the surrounding fluid. The analytical signal-tonoise ratio is derived, yielding a direct physical insight with respect to the system design. Experimental behaviors are very well reproduced, and numerical finite elements methods are implemented to successfully confirm the relevance of our approach. We also provide a detailed explanation of the coupling dynamics between the quartz tuning fork and the acoustically resonant tube.
\end{abstract}

\section{Introduction}

Photoacoustic spectroscopy (PAS) is one of the most sensitive techniques for trace gas measurements. It addresses a wide range of demanding applications such as environmental or industrial monitoring, pollution control or medical breath analysis [1, 2]. For decades, conventional PAS has been employed by combining three elements: a laser source, an acoustic cavity and a standard microphone, as depicted in Fig. 1. It is now well understood and accurate analytical theoretical descriptions have been developed [3]. If the acoustic cavity is operated at one of its resonance frequencies, the PAS technique is qualified as resonant.

Guillaume Aoust

guillaume.aoust@mirsense.com

1 ONERA-The French Aerospace Lab, 91123 Palaiseau cedex, France

2 mirSense, Nano-INNOV, 91120 Palaiseau, France
In 2002, Kosterev et al. proposed to replace the standard microphone with a high-quality-factor resonator such as a commercial quartz tuning fork [4]. The latter sensing element is very small and cheap. Moreover, early measurements showed a very promising potential, with a relatively good immunity to ambient noise. Similar to conventional PAS, this new configuration is referred as "quartz-enhanced photoacoustic spectroscopy" (QEPAS) when a bare quartz tuning fork is used, and "resonant QEPAS" if elements are added to benefit from an acoustic resonance. Usually, the acoustic resonator consists of hollow tubes, and the tuning fork is placed at one of the openings. These tubes can greatly enhance the signal to noise ratio, up to a factor of 30 in the on-beam configuration [5-7]. We recall in Fig. 2 some of the used configurations. For more details, the reader should report to a recent review of the technique available in [8].

In any case, the technique has been mainly experimentally investigated so far, and very little analytical theoretical investigation has been provided. Indeed, none of the previous models developed for conventional PAS is valid. It is also worthwhile to note that tuning forks have also been used in a very similar setup called Resonant OptoThermoAcoustic DEtection (ROTADE) [9]. Instead of the acoustic waves generated by the photoacoustic effect, ROTADE focuses on the heat diffusion thermal waves induced by the absorbed radiation. However, ROTADE modelling cannot be applied to QEPAS since the two techniques rely on a very different physics. The former deals with heat propagation and constraints withing the tuning fork [10] while the latter, as we will see in the following, consists mainly of a vibroacoustic problem.

A first analytical model for QEPAS has been developed by Petra et al. in the case of a bare tuning fork [11]. Neglecting the influence of the mechanical resonator on 
Fig. 1 Principle of conventional photoacoustic spectrometry (conventional PAS). The molecular mix is usually flowing through a cell, with a microphone fixed on its walls. When the modulation frequency matches an eigenfrequency of the cell, the technique is referred as resonant conventional PAS

Fig. 2 Schematic of existing configurations of resonant QEPAS. The tuning fork is positioned close to an opening of the acoustic resonant cavities, which are here in the form of hollow tubes

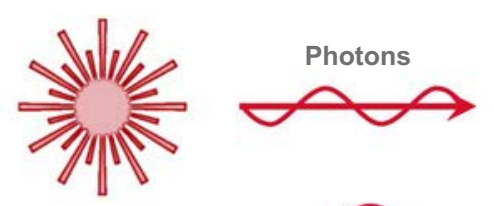

1

Modulated light

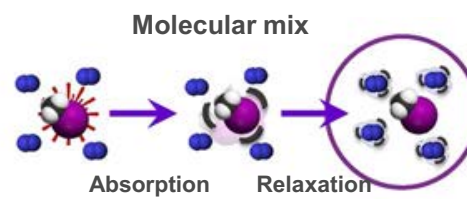

2
Selective Absorption Relaxation
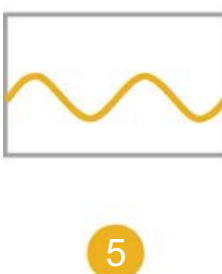

Electrical processing

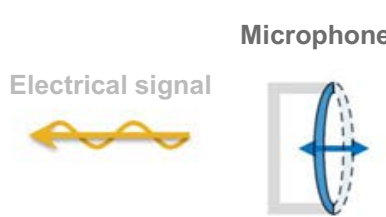

4

Acoustic waves detection

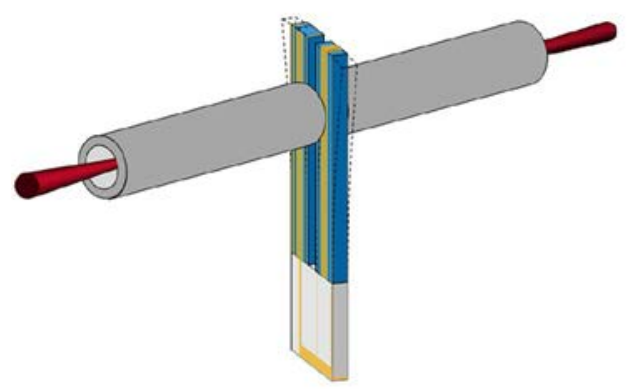

On-Beam QEPAS

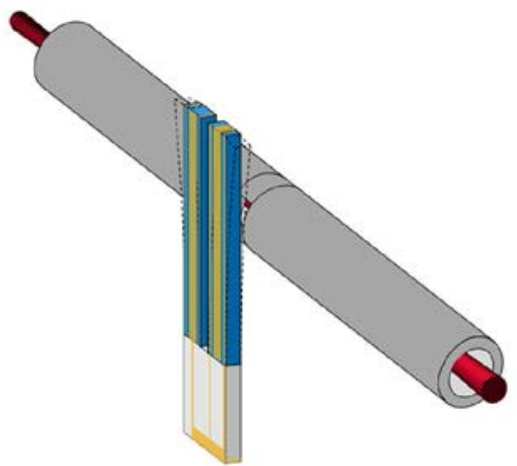

Off-Beam QEPAS
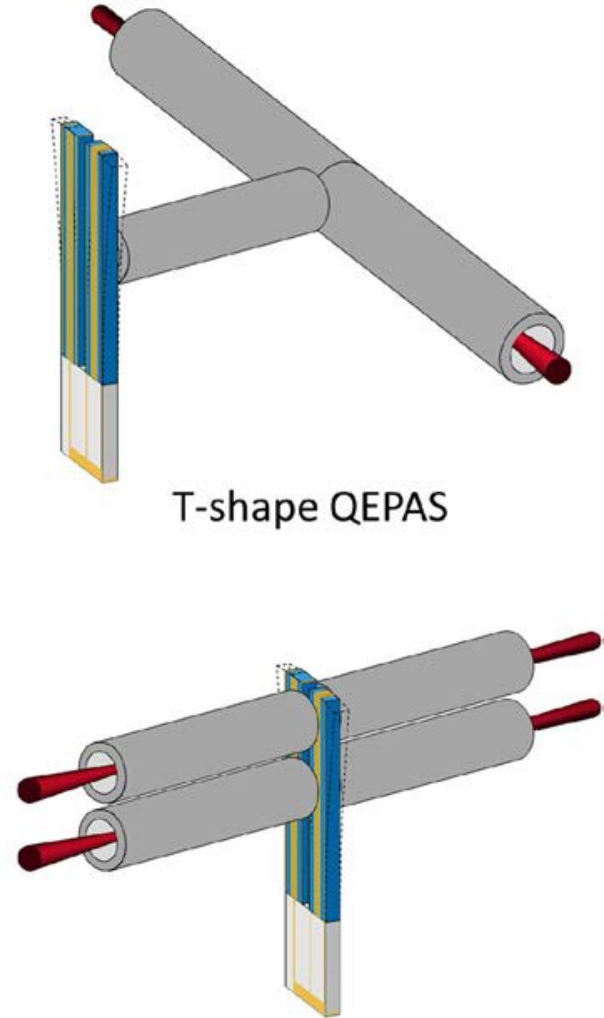

Multi-Beam QEPAS the pressure field created by the photoacoustic effect, their results reproduce well the variation of the signal as a function of the laser position between the prongs. The calculated signal-to-noise ratio shows reasonable agreement with experiments. However, a factor of two between theory and experiment is still unexplained. Furthermore, their model does not take into account any possible additional acoustic resonator nearby; the modeling of the most sensitive QEPAS experiments thus remains impossible. 
Besides, an additional physical phenomenon reported in the literature cannot be described by this first model. Indeed, experiments have shown that an anti-resonance phenomenon occurs for specific acoustic cavity geometries, leading to dramatic modifications of the tuning forks resonance features $[5,12]$. It is a clear indication that a vibroacoustic interaction does occur in this case, and thus the generation of the acoustic field should not be considered as independent from the mechanical resonator. In this context, an analytical treatment has been proposed to investigate the acoustic resonator optimum dimensions [13], but this model neglects the vibro-acoustic coupling and it only gives the influence of the acoustic quality factor on the QEPAS signal.

An analytical solution to the complete problem is tricky, because it leads to a system of coupled equations. Moreover, it involves multiphysics phenomena which are difficult to handle simultaneously. For these reasons, multiphysics modeling of resonant QEPAS experiments has only been carried out by use of numerical simulations so far [14-17]. For example, Cao et al. numerically investigated the influence of the length and inner diameter of two acoustic tubes used in an on-beam configuration
[16]. Their result shows complex behaviors for which the influence of each parameter involved in the setup seems hard to determine.

In this article, we develop an original analytical model for resonant QEPAS taking into account the acoustic resonator and its coupling with the mechanical resonator. First, we formalize a simplified vibroacoustic problem using a new set of assumptions, hence leading to a single equation generalizing the known fundamental equation for photoacoustics. We then compare the obtained analytical results with numerical simulations as well as laboratory experiments.

\section{Analytical description of resonant QEPAS}

Most of the time, a resonant QEPAS experiment uses two acoustic tubes and a quartz tuning fork, in an on-beam configuration, as detailed in Fig. 3. For the sake of simplicity, our study will focus on this practical case, even if our results can be generalized to any acoustic configuration and any mechanical resonator.

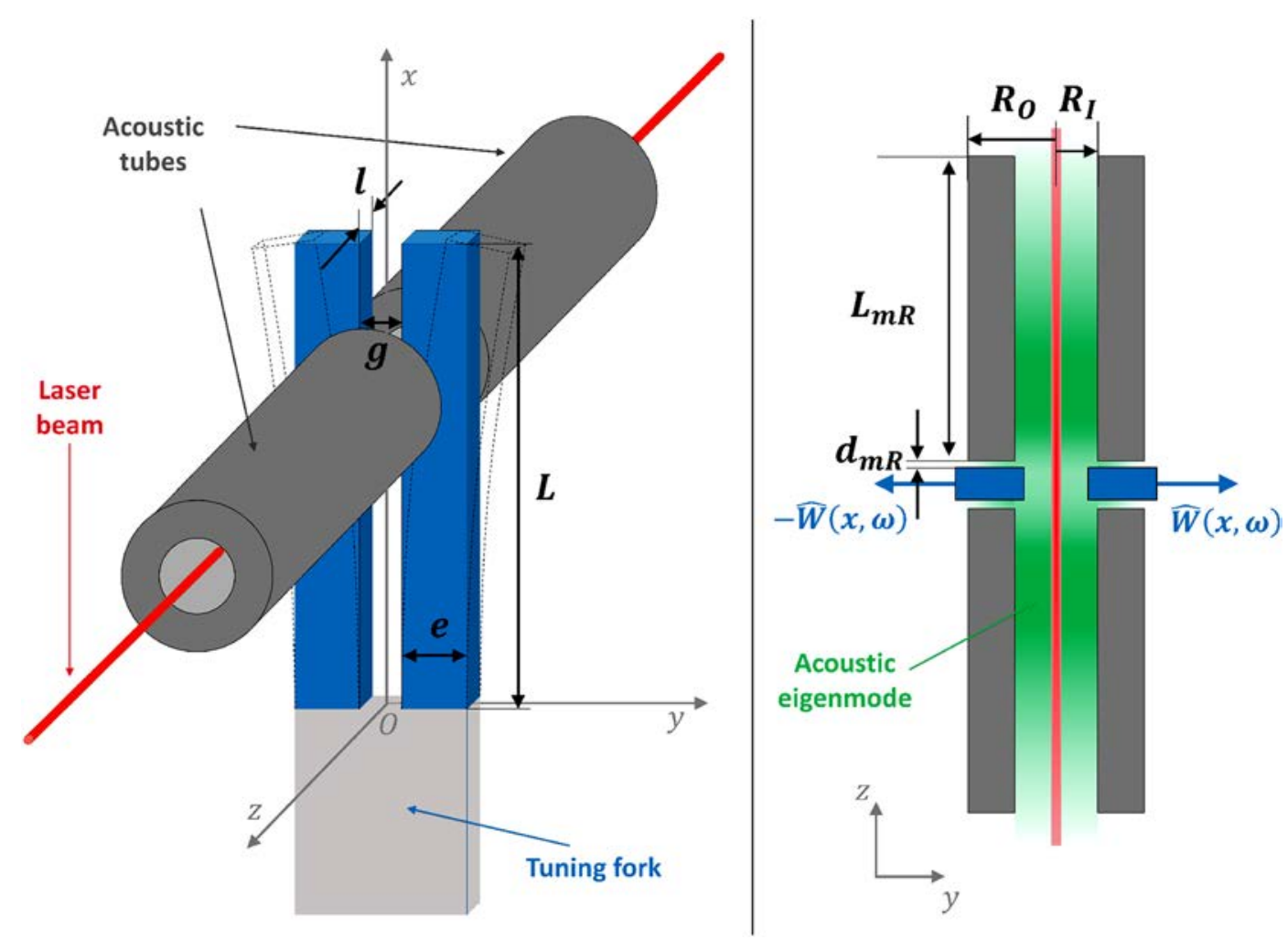

Fig. 3 Schematic of an on-beam configuration of QEPAS. The tuning fork is inserted in the middle of a hollow tube, which hosts an acoustic resonance. This acoustic resonance is excited by the photoacoustic effect generated along the tube by the laser beam, which occurs if the laser source wavelength is in coincidence with an absorption feature of the gas molecule in the tube. The tuning fork is excited by the acoustic resonance acting on the tuning fork prongs 


\subsection{Problem formulation}

\subsubsection{Definitions and notations}

We will first assume that the laser beam is centered between the prongs. To formalize the situation, we introduce the following notations in agreement with Fig. 3:

- $e, l$ and $L$ are, respectively, the prongs width, thickness and length.

- $g$ is the gap between the two prongs.

- $\rho_{\mathrm{p}}$ is the volumetric mass density of the prongs.

- $\hat{W}$ is the displacement of the tuning fork in the $y$ direction.

- $v$ is the speed of sound in the medium and $\rho_{\mathrm{f}}$ is the fluid volumetric mass density.

The origin of the coordinate system is chosen such that the inner surface of the prongs is located at $y= \pm g / 2$, $-l / 2<z<l / 2$ and $0<x<L$.

During a QEPAS experiment, the tuning fork is excited close to one of its eigenmodes angular frequencies $\omega_{n}$ . The displacement of each prong in the $y$ direction can then be modeled as a second- order low-pass filter [18]:

$\hat{W}(x, \omega)=\hat{w}(\omega) \phi_{n}(x)=\frac{F_{n}}{\left[K_{n}+j \eta_{n} \omega-M_{n} \omega^{2}\right]} \phi_{n}(x)$

In Eq. (1), $F_{n}, K_{n}, \eta_{n}$ and $M_{n}$ are, respectively, the resonator equivalent excitation force, stiffness, damping and mass. Function $\phi_{n}$ is the $n$th normalized deformation of the prongs, and its expression can be found in any mechanical textbook (e.g. [19]). The equivalent coefficients are linked to the tuning fork actual geometric characteristics, and the equivalent excitation force is linked to the pressure $\hat{P}$ exerted on the prongs surfaces:

$\left\{\begin{array}{l}M_{n}=\rho_{\mathrm{p}} e l \int_{0}^{L} \phi_{n}^{2}\left(x^{\prime}\right) \mathrm{d} x^{\prime} \\ K_{n}=\omega_{n}^{2} M_{n} \\ \eta_{n}=\frac{\omega_{n} M_{n}}{Q} \\ F_{n}=\int_{0}^{L} \int_{-l / 2}^{l / 2}\left[\hat{P}\left(x^{\prime}, \frac{g}{2}, z, \omega_{n}\right)-\hat{P}\left(x^{\prime}, \frac{g}{2}+e, z, \omega_{n}\right)\right] \phi_{n}\left(x^{\prime}\right) \mathrm{d} z \mathrm{~d} x^{\prime}\end{array}\right.$ [21]:
We also introduced in Eq. (2c) the quality factor of the bare tuning fork $Q$ (the quality factor without any acoustic resonator nearby), which is typically of $10^{4}$ for commercially available quartz tuning forks vibrating in air on their fundamental mode of vibration. If necessary, an analytical expression can be found in the literature, as a function of the tuning fork geometry and the surrounding fluid $[18,20]$. In particular, the latter articles show that
$Q$ can be quantitatively predicted quite accurately with a fully analytical formula (typically within 10\%).

\subsubsection{QEPAS vibroacoustic problem formulation}

Using the Fourier domain, we introduce the wavenumber $k_{v}=\omega / v$. It is known that the pressure field $\hat{P}$ at position $\mathbf{r}$ in $3 \mathrm{D}$ space is the solution to the Helmholtz equation with boundary conditions imposed by the surfaces nearby

$\begin{cases}\nabla^{2} \hat{P}(\mathbf{r}, \omega)+k_{v}^{2} \hat{P}(\mathbf{r}, \omega) & =-j \omega \rho_{\mathrm{f}} \hat{q}(\mathbf{r}, \omega) \\ (\vec{\nabla} \hat{P}(\mathbf{r}, \omega)) \cdot \vec{n} & =\left(\rho_{\mathrm{f}} \omega^{2} \hat{W}(\mathbf{r}, \omega) \vec{y}\right) \cdot \vec{n} \\ \lim _{|\mathbf{r}| \rightarrow+\infty}|\mathbf{r}|\left(\frac{\partial}{\partial|\mathbf{r}|}+j k_{v}\right) \hat{P}(\mathbf{r}, \omega) & =0\end{cases}$

The first Eq. (3a) is sometimes called the fundamental equation of photoacoustics [3] and is valid within the fluid. The volume flow density $\hat{q}(\mathbf{r}, \omega)$ is linked to the heat density $\hat{H}$ deposited in the gaz. In the absence of any additional source of sound, for example in the "classical" formulation of the photoacoustic effect [3], its expression is given as follows using the surrounding gas heat capacity ratio $\gamma$ :

$\hat{q}(\mathbf{r}, \omega)=\frac{\gamma-1}{\rho_{\mathrm{f}} v^{2}} \hat{H}(\mathbf{r}, \omega)$

Equation (3b) is the boundary condition at the interface between the fluid and any solid, with $\vec{n}$ a vector normal to the surface of the solid. It represents the impact of any moving solid on the fluid pressure. The solid can either be the tuning fork or the acoustic resonator. It turns out that the right-hand term of the equation always vanishes except when the solid is moving along $\vec{n}$. The latter condition only happens for the tuning fork prongs, and the gradient of the pressure is then linked to the prongs displacement $\hat{W}$ which

we assumed to be in the $\vec{y}$ direction. The third Eq. (3c) is known as the Sommerfeld radiation condition and sets the boundary condition far from the resonator.

The vibroacoustic interaction is taken into account in this system, contrary to previous models [11]. The prongs movement $\hat{W}$ is generated by the pressure field $\hat{P}$, which in turn modifies the pressure field through the boundary condition (3b). 


\subsection{The resonant QEPAS fundamental equation}

\subsubsection{The acoustic source equivalence}

We now use a slightly simpler formulation for this vibroacoustic problem. For that purpose, we replace the boundary condition ( $3 b$ ) imposed by the tuning fork movement by an equivalent acoustic source, as shown in Fig. 4.

A tuning fork can indeed be modeled as an acoustic longitudinal quadrupole, hence we will use four $y_{0}$ locations to describe its acoustic emission: $y_{0}= \pm g / 2$ and $y_{0}= \pm(g / 2+e)$ [22]. A new contribution for each point has to be added to the volume flow density $\hat{q}$. More details can be found in our previous work related to the acoustic emission of high- quality resonators [20].

Before writing our final system, we define an extended normalized deformation function valid in all 3D space and denoted $\Phi_{n}(x, y, z)$. The idea is to use the normalized deformation function $\phi_{n}$ in the whole space. Its values are $\phi_{n}(x)$ on the inner and outer moving surfaces of the prongs, and zero elsewhere. In mathematical terms, we can use the indicator function $\mathbb{1}$ and $\delta$ as the Dirac function to define it:

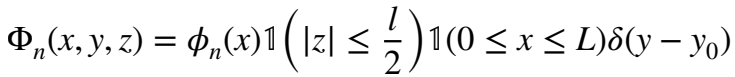

Adding the tuning fork movement described by Eq. (1) to the fluid problem set of equations $(3 a, 3 b, 3 c)$, we obtain the resonant QEPAS fundamental equation, where the boundary condition ( $3 \mathrm{~b}$ ) is replaced by the equivalent quadrupole acoustic source: a Tuning fork section

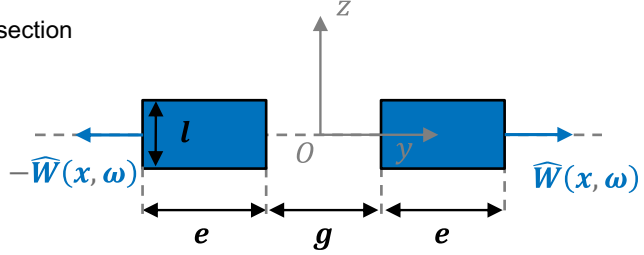

b Acoustic equivalent longitudinal quadrupole

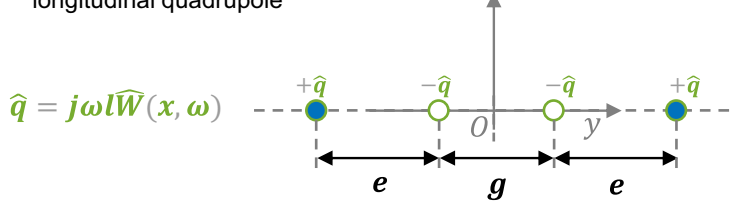

Fig. 4 Acoustic equivalent of a tuning fork section oscillating in a fluid medium. The prongs movement can be acoustically replaced by volume flow densities $\hat{q}$, located at the faces that are perpendicular to the direction of movement, hence forming a longitudinal quadrupole

\subsubsection{The resonant QEPAS solution}

Since an analytical solution is difficult to find, we make here an additional assumption about the pressure field. We assume that the excitation frequency is close to the eigenfrequency of one of the pressure eigenmodes in the absence of mechanical motion. These pressure eigenmodes and their corresponding eigenfrequencies can be obtained by solving the Helmholtz equation (6a) under the assumption $\hat{q}=0$. The considered boundary conditions $(3 b)$ and $(3 c)$ remain unchanged. It can be noted here that numerical simulations are generally very well suited to obtain the pressure eigenmodes. We can now write the pressure field as follows:

$$
\begin{cases}\nabla^{2} \hat{P}(x, y, z, \omega) & +k_{v}^{2} \hat{P}(x, y, z, \omega)=-j \omega \rho_{\mathrm{f}} \hat{q}(x, y, z, \omega) \\ \hat{q}(x, y, z, \omega) & =\frac{\gamma-1}{\rho_{\mathrm{f}} \nu^{2}} \hat{H}(x, y, z, \omega)+2 j \omega \hat{w}(\omega)\left[\Phi_{n}\left(x, \frac{g}{2}+e, z\right)-\Phi_{n}\left(x, \frac{g}{2}, z\right)\right] \\ \hat{w}(\omega) & =\frac{\int_{0}^{L}\left[\int_{-l / 2}^{l / 2}\left[\hat{P}\left(x, \frac{g}{2}, z, \omega_{n}\right)-\hat{P}\left(x, \frac{g}{2}+e, z, \omega_{n}\right)\right] \phi_{n}(x) d z\right] \mathrm{d} x}{\left[K_{n}+j \eta_{n} \omega-M_{n} \omega^{2}\right]} \\ 0 & =\lim _{|\mathbf{r}| \rightarrow+\infty}|\mathbf{r}|\left(\frac{\partial}{\partial|\mathbf{r}|}+j k_{v}\right) \hat{P}(\mathbf{r}, \omega)\end{cases}
$$

Keeping the terms due to the acoustic emission of the mechanical resonator, we assume that the acoustic emission induced by the tuning fork movement cannot be neglected. It fundamentally differs from the previous analytical work of Petra et al. [11], which assumes that the presence of the tuning fork does not affect the acoustic field generated by the photoacoustic effect. We hence obtain an analytical situation very close to that numerically obtained with finite element simulation software, with the tuning fork acoustic re-radiation taken into account [15].
$\hat{P}(\mathbf{r}, \omega)=A_{m}(\omega) \hat{P}_{m}(\mathbf{r})$

Similar to the work of Miklós et al. for classical acoustic cavities, we introduce $\omega_{m}$ the pressure eigen-angular frequency as well as $Q_{a}$, the quality factor, to take into account the losses of the acoustic system (without any tuning fork movement) [3].

Using Eq. (7) in system (6a, 6b, 6c, 6d), we obtain:

$$
\begin{aligned}
A_{m}(\omega)\left[\omega_{m}^{2}-\omega^{2}+\right. & \left.j \omega \omega_{m} Q_{a}^{-1}\right] \hat{P}_{m}(x, y, z)=j \omega(\gamma-1) \hat{H}(x, y, z, \omega) \\
& +2 \omega^{2} \rho_{\mathrm{f}} v^{2} \hat{w}(\omega)\left[\Phi_{n}\left(x, \frac{g}{2}, z\right)-\Phi_{n}\left(x, \frac{g}{2}+e, z\right)\right]
\end{aligned}
$$


The heat density $\hat{H}(\mathbf{r}, \omega)$ can be easily expressed if the vibrational-translational characteristic time $\tau_{V-T}$ of the absorbing gas satisfies $\omega \tau_{V-T}<<1$, in the common case of a modulated laser intensity at a fixed wavelength:

$\hat{H}(\mathbf{r}, \omega)=\alpha_{G} P_{L}|u(r)|^{2}$

In Eq. (9), $\alpha_{G}$ denotes the gas absorption per unit length at the laser wavelength, $P_{L}$ the laser power and $|u(r)|^{2}$ the normalized intensity distribution (its integral over the entire cross section of the laser beam is normalized to unity). We define what is called the "normalized overlap integral" by the following formula:

$O_{m}=\frac{\frac{1}{L_{t}} \int|u(r)|^{2} \hat{P}_{m}^{*}(r) \mathrm{d} V}{\frac{1}{V_{\text {cell }}} \int\left|\hat{P}_{m}(r)\right|^{2} \mathrm{~d} V}$

This factor represents the overlap between the laser source and the acoustic eigenmode, normalized to its total length of interaction $L_{t}$. Length $L_{t}$ is the adjusted length of the acoustic cavity along the laser propagation direction, taking into account the "end corrections" [3]. Physically, the end corrections take into account the mismatch between the one-dimensional acoustic field inside the tubes and the three-dimensional field outside that is radiated by the open ends. The value $V_{\text {cell }}$ is the total volume of the resonant cavity. Denoting $F_{n m}$ the modal force acting on the quartz resonator, it can be expressed as

$F_{n m}=\int_{0}^{L} \int_{-l / 2}^{l / 2}\left[\phi_{n}(x)\left(\hat{P}_{m}(x, d, z)-\hat{P}_{m}(x, d+e, z)\right)\right] \mathrm{d} x \mathrm{~d} z$.

We can then introduce a new coefficient called "normalized vibroacoustic mode coupling integral", denoted $C_{n m}$, and defined as follows:

$C_{n m}=\frac{\left[\frac{1}{l L}\left|F_{n m}\right|\right]^{2}}{\frac{1}{V_{\text {cell }}} \int\left|\hat{P}_{m}(r)\right|^{2} \mathrm{~d} V}$

The two latter coefficients $O_{m}$ and $C_{n m}$ can be used to write the evolution of the amplitude of the pressure, by integrating Eq. (6a) over the cavity volume:
$\hat{W}(x, \omega)=\hat{w}(\omega) \phi_{n}(x)=\frac{A_{m}(\omega) F_{n m}}{\left[K_{n}-M_{n} \omega^{2}+j \omega \eta_{n}\right]} \phi_{n}(x)$

In the case of a negligible normalized vibroacoustic mode coupling integral $\left(C_{n m}=0\right)$, we retrieve naturally the same result for the pressure as Miklós et al. [3].

\subsubsection{Signal to noise ratio}

It is now well established that the ultimate noise source for a QEPAS experiment is the Brownian noise of the quartz resonator [23]. This noise is equivalent to a white equivalent force acting on the tuning fork in the bare QTF case, whose expression is given as follows:

$F_{b}(\omega)=\sqrt{4 k_{B} T \eta_{n} \Delta f}$,

where $k_{B}$ is the Boltzmann constant, $T$ the tuning fork temperature and $\Delta f$ is the detection bandwidth. We assume that this modeling of noise is still valid in the resonant QEPAS case, under the condition that the quality factor used in $\eta_{n}$ coefficient (see Eq. 2c) is the one observed on the tuning fork behavior (which is potentially modified by the acoustic resonator because of the vibroacoustic coupling). The equivalent noise displacement of the tuning fork can hence be written as follows:

$\hat{W}_{b}(x, \omega)(\omega)=\frac{\sqrt{4 k_{B} T \eta_{n} \Delta f}}{\left[K_{n}-M_{n} \omega^{2}+j \omega \eta_{n}\right]} \phi_{n}(x)$

The signal to noise ratio can hence be simply obtained:

$\mathrm{SNR}=\sqrt{2}\left|\frac{\hat{W}}{\hat{W}_{b}}\right|=\sqrt{2}\left|\frac{A_{m}(\omega) F_{n m}}{\sqrt{4 k_{B} T \eta_{n} \Delta f}}\right|$

The signal generated by each prong is proportional to the displacement, according to the laws of piezoelectricity. The charges collected on each prongs by the electrodes are added constructively, and the signal generated by a tuning fork is hence doubled compared to that of a single prong. The two noises, however, only add in quadratic mean since they are uncorrelated. The two latter remarks explain the

$A_{m}(\omega)=\frac{j \omega(\gamma-1)\left[K_{n}-M_{n} \omega^{2}+j \omega \eta_{n}\right] L_{t} O_{m} \alpha_{G} P_{L}}{\left[\omega_{m}^{2}-\omega^{2}+j \omega \omega_{m} Q_{a}^{-1}\right]\left[K_{n}-M_{n} \omega^{2}+j \omega \eta_{n}\right] V_{\text {cell }}-2 \omega^{2} \rho_{\mathrm{f}} \nu^{2} l^{2} L^{2} C_{n m}}$

The pressure inside the cavity is still given by Eq. (7), and can be substituted in the displacement expression (Eq. 1). We finally obtain the motion of the tuning fork, solution to the resonant QEPAS problem: $\sqrt{2}$ factor in Eq. (17), and is specific to the use of a tuning fork compared to a single prong or cantilever. 


\section{Experiments on resonant QEPAS}

In this section, we investigate the validity of our analytical model using a numerical simulation software as well as laboratory experiments. In particular, we study the influence of the acoustic tube length on the tuning fork behavior.

We consider an on-beam QEPAS setup with tuning fork dimensions measured as $e=0.6 \mathrm{~mm}, l=0.34 \mathrm{~mm}$, $L=3.75 \mathrm{~mm}$ and $g=0.31 \mathrm{~mm}$. Our optical source is a commercial laser diode enabling a wavelength tuning range from 6487 to $6494 \mathrm{~cm}^{-1}$. The height $h$ of the beam between the prongs is located $0.6 \mathrm{~mm}$ below the top opening $(h=L-0.6 \mathrm{~mm})$.

We use a certified mixture of $2.7 \% \mathrm{CO}_{2}$ in $\mathrm{N}_{2}$, and we add water vapor with $15 \%$ relative humidity. The presence of water allows a faster relaxation of carbon dioxide, and we assume that the relaxation rate $\tau_{V-T}$ is negligible [24]. The density of the mixture is taken as $\rho_{\mathrm{f}}=1.2 \mathrm{~kg} \mathrm{~m}^{-3}$ while the sound velocity is $v=346 \mathrm{~m} \mathrm{~s}^{-1}$. We consider the $\mathrm{CO}_{2}$ absorption peak located at $k_{G}=6490.05 \mathrm{~cm}^{-1}$, which displays an absorption per unit length of $\alpha_{G}=510^{-6} \mathrm{~cm}^{-1}$ according to the HITRAN database [25].

We define the total quality factor $Q_{t}$ of a resonant QEPAS setup as the $3 \mathrm{~dB}$ bandwidth measured on its frequency response. This definition is commonly used in actual experiments, because the assumed resonant behavior is that of a second-order linear system. This choice could, however, be questioned, because we showed that resonant QEPAS is closer to a fourth-order linear system because of the acoustic resonance (see Eq. 14). The resonance frequency $f_{\text {res }}$ is defined as the frequency maximizing the prongs displacement $\hat{W}$. Since the displacement of the prongs is proportional to the electric signal generated by the tuning fork, any reasoning about the prongs displacement is interchangeable with the signal produced by the tuning fork and detected in a QEPAS experiment.

\subsection{Numerical experiments}

We used the commercially available finite element simulation software OOFELIE::Multiphysics@ to obtain numerical results of our system behavior [26, 27]. We assume a typical laser beam radius at $1 / \mathrm{e}^{2}$ of $w_{L}=50 \mu \mathrm{m}$, and we neglect diffraction causing the beam radius to change with propagation. The power of the laser is supposed to be sine modulated at the fundamental resonance frequency $f_{0}$ of the tuning fork, with a realistic average power of $P_{L}=17 \mathrm{~mW}$. Neither fluid viscosity nor temperature effects are taken into account in this simulation. We choose, however, a quartz structural damping of $Q=10^{4}$ to account for the actual damping acting on the tuning fork caused by gas viscosity.

We show in Fig. 5 a typical simulation using tubes with a length of $L_{m R}=6.7 \mathrm{~mm}$, an inner radius of $R_{\mathrm{I}}=0.46$ $\mathrm{mm}$, an outer radius of $R_{\mathrm{O}}=0.64 \mathrm{~mm}$ and a distance of $d_{m R}=20 \mu \mathrm{m}$ from the tuning fork prongs. The latter notations have already been illustrated in Fig. 3. The excitation frequency is chosen close to the tuning fork fundamental eigenfrequency.

The simulation clearly confirms the resonant nature of the acoustic field. The presence of the acoustic eigenmode is almost exclusively limited to the inner volume of the tubes and the space between the two prongs. We can also see that the acoustic eigenmode has no dependence along the tube radius, which is consistent with the acoustic wavelength $\lambda_{v} \approx 10.6 \mathrm{~mm}$ being much greater than the
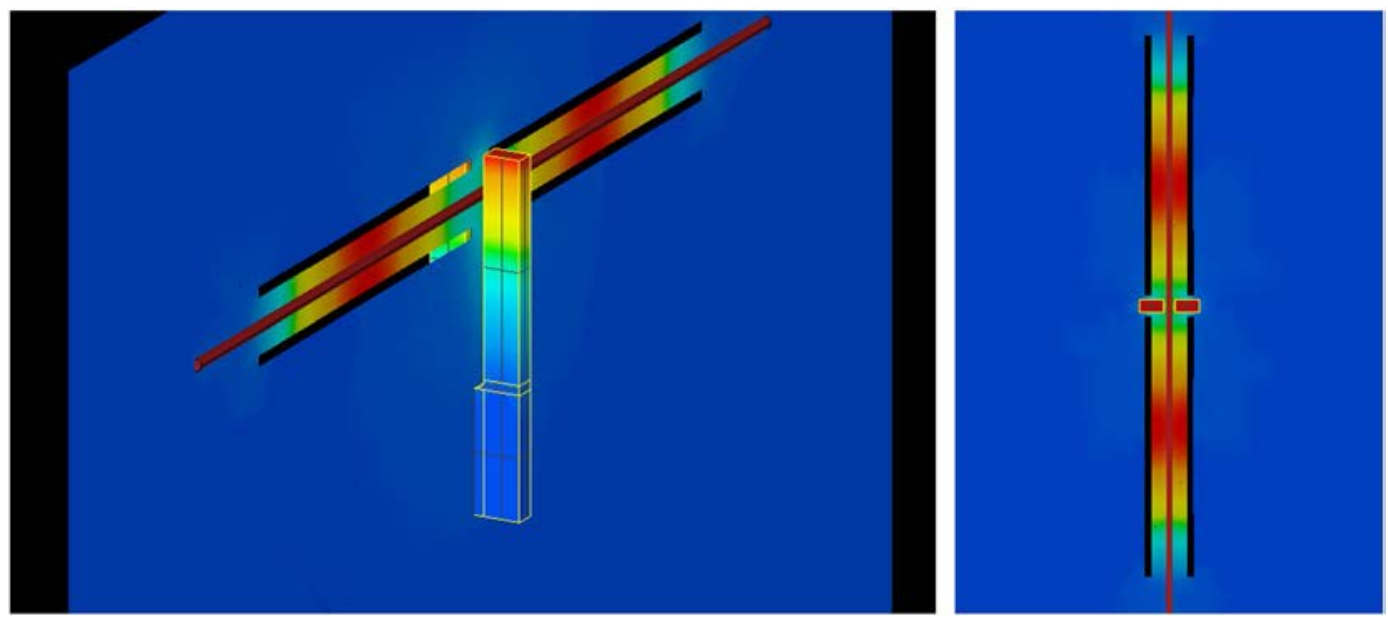

Fig. 5 Amplitude of the pressure field for two observation planes $y=0$ (left) and $x=h$ (right, seen from above the tuning fork), for a tube length of $L_{m R}=6.7 \mathrm{~mm}$ and inner radius $R_{\mathrm{I}}=0.46 \mathrm{~mm}$. The linear color scale is normalized, ranging from blue to red. An independent color scale is also applied to the tuning fork to visualize the displacement 

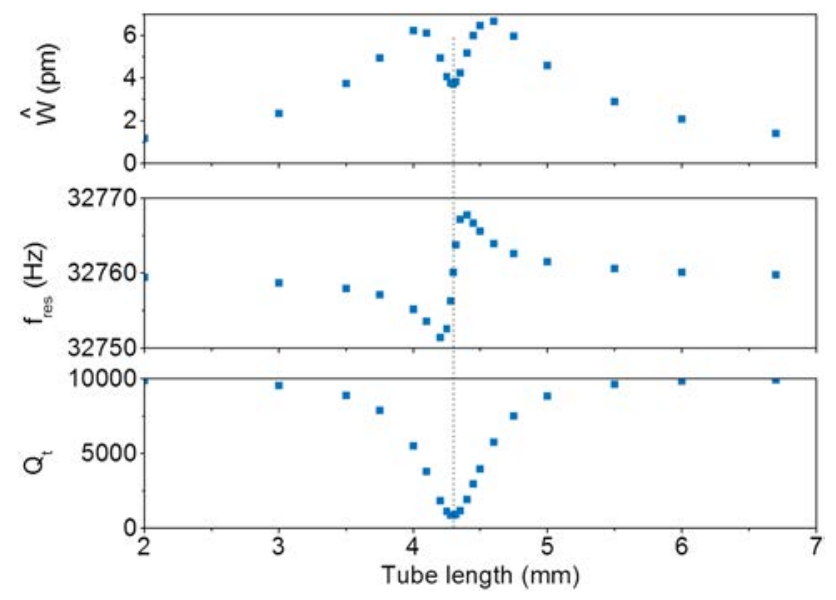

Fig. 6 Influence of the length $L_{m R}$ of the acoustic tubes, obtained with the numerical simulation software OOFELIE. The inner diameter of the tubes is $R_{\mathrm{I}}=0.46 \mathrm{~mm}$ while the outer diameter is $R_{\mathrm{O}}=0.64 \mathrm{~mm}$

tube inner radius $R_{\mathrm{I}}$. Keeping exactly the same tube positioning with respect to the tuning fork and only varying its length, we obtain the results shown in Fig. 6. For each tube length, a simulation is used to describe the resonance of the system, by sweeping the modulation frequency of the laser across the resonance (which is always close the tuning fork resonance frequency in the absence of acoustic tubes). Results are refined if the obtained curve is not sufficiently sampled. At the end of this process, we precisely know the resonance frequency of the system, the maximum displacement of the prongs as well as the total quality factor of the system by measuring the $3 \mathrm{~dB}$ bandwidth.

The tuning fork behavior strongly depends on the tube's length according to this simulation. The latter result is very similar to that obtained by Cao et al. [16], who used COMSOL multiphysics commercial software. The electrical signal generated by the tuning fork, which is proportional to the prongs displacements $\hat{W}$ reported in Fig. 6, presents what is called an antiresonance for a specific length $\left(L_{m R}=4.3 \mathrm{~mm}\right.$ in our case). This antiresonance is attributed to the exact superposition of the resonance frequencies of the tuning fork on the one hand and of the acoustic resonant mode on the other hand.

The acoustic quality factor of the cavity $Q_{a}$, representing the amount of losses of the acoustic resonant cavity, is only determined by the radiations at the tubes opening in this numerical model. Indeed, our simulation neglects viscous and thermal effects that occur, even if these effects can have a major impact in an actual experiment [28]. We will discuss this point in further details in the experimental Sect. 3.3, but we stress here that the quality factor $Q_{a}$ computed by the current simulation can hence vary with the length of the tubes. Its average value is found to be $Q_{a}=30$ when the tube lengths vary from 2 to $7 \mathrm{~mm}$, determined using a numerical simulation with a fully clamped tuning fork.

\subsection{Analytical model prediction}

\subsubsection{Parameters adjustments}

Before applying our results obtained in the theoretical Sect. 2, we need more information about the acoustic field. The previous results in Fig. 5 confirmed the resonant nature of the acoustic field, which is an important assumption we made in the analytical model. To compare the numerical results with our analytical expression, we need an expression of the acoustic distribution $\hat{P}_{m}$ to compute the two overlap factors $O_{m}$ and $C_{n m}$. It could be done using the numerical simulation results since the exact distribution is complex to describe analytically. We nevertheless choose to make additional assumptions preserving the physical insight to obtain an analytical expression.

We call the "resonant cavity" the set composed of the inner space between the prongs as well as the inner volume of the tubes. Its total length is hence $L_{t}=2\left(L_{m R}+\Delta L_{m R}+d_{m R}+l / 2\right)$, and its volume is $V_{\text {cell }}=2 \pi R_{I}^{2}\left(L_{t}-l\right)+g L l$. The quantity $\Delta L_{m R}$ is the "end correction", and is generally approximated by $\Delta L_{m R} \approx 0.6 R_{I}$ for a tube termination opening in a semiinfinite space [3]. We also assume that the amplitude of the pressure is negligible outside of the resonant cavity. The motion of the resonator is hence only due to the action of the acoustic eigenmode pressure forces on its inner surfaces. The distance between the two prongs $g$ as well as the inner radius of the tubes $R_{I}$ is very small compared to the acoustic wavelength $\left(\lambda_{v}=10.6 \mathrm{~mm}\right.$ in our case $)$, the resonant cavity can hence be seen as a one-dimensional resonator and the acoustic distribution does not depend on the $y$ and $x$ variables. In addition, the thickness $l$ is also small compared to $\lambda_{v}$, and we will hence consider the pressure as a constant between the prongs. The Helmholtz equation (6a) now only depends on the $z$ variable, and the solution can be written as follows:

$\hat{P}_{m}(z)=\sin \left[\frac{\omega_{m}}{v}\left(\frac{L_{t}}{2}-|z|\right)\right]$

In the previous expression, the acoustic angular eigenfrequency $\omega_{m}$ considered is the one closest to $\omega_{n}$, that is for $m=2$ and reads:

$\omega_{2}=2 \pi \frac{v}{L_{t}}$

In addition, we introduced in our formalism the quality factor $Q_{a}$ of the resonant cavity (Eq. 8). It is known that this value depends on the acoustic radiation losses of the cavity, as well as the viscosity and thermal properties of the fluid 
[29]. We will consider the same quality factor as the one computed with the previous numerical simulations, that is $Q_{a}=30$. Since we use the approximate expression (Eq. 18) for the pressure distribution, we allow an adjustment on the critical $C_{n m}$ coefficient. We will decrease its original value of $510^{-3}$ (Eq. 12) down to $210^{-4}$, a value chosen to match the overall quality factor drop observed in the numerical simulation. We remind that this parameter remains the same for any length $L_{m R}$ and does not affect the shape of the curves.

\subsubsection{Results and discussion}

Analytical results obtained with the adjusted $C_{n m}$ coefficient are shown in Fig. 7.

The agreement between the finite element numerical simulation and the analytical model is remarkable. Every aspect of the resonance is correctly reproduced by the model, namely the varying resonance frequency, the drop shape of the overall quality factor $Q_{t}$ and the singular antiresonant shape of the signal curve. The value of the tube length $L_{m R}$ that maximizes the quality factor drop is slightly different $(4.5 \mathrm{~mm}$ here versus $4.2 \mathrm{~mm}$ with the finite element modeling), which can be explained by the use of an approximate expression for the eigen-angular frequency (19). This discrepancy is usually addressed with the use of the end corrections, but, in our case, we would need an additional end correction representing the radiation losses occurring where the tuning fork is inserted.

According to the analytical prediction, the amplitude of the prongs displacement is halved compared to the numerical prediction. The discrepancy can be attributed to the

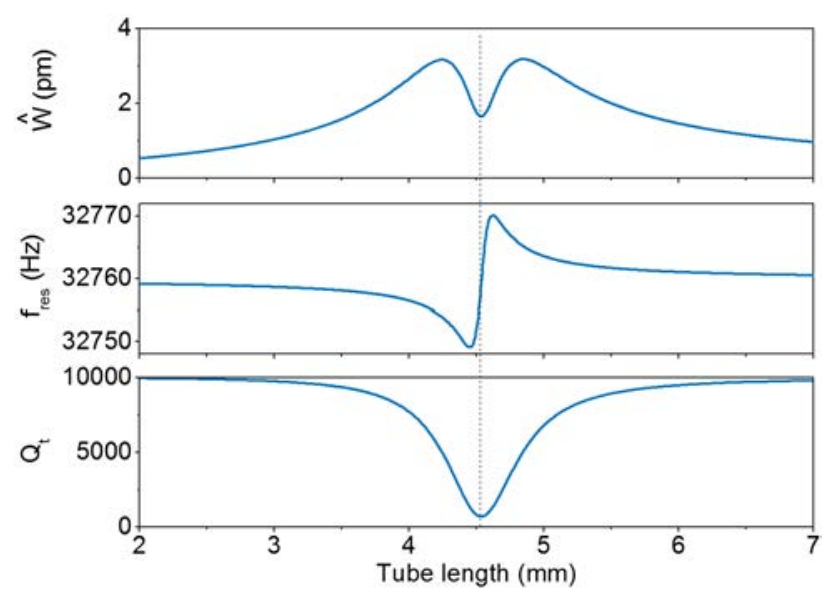

Fig. 7 Influence of the tube length $L_{m R}$ for our on-beam QEPAS experiment, computed using our analytical model. The inner diameter of the tubes is $R_{\mathrm{I}}=0.46 \mathrm{~mm}$ while the outer diameter is $R_{\mathrm{O}}=0.64 \mathrm{~mm}$ simplicity of the model used for the pressure eigenmode spatial distribution.

The drop in the quality factor of the resonant QEPAS has already been reported in the literature [12]. We will also confirm that observation in our experimental section. This drop is the characteristic of the vibroacoustic coupling, and we notice that the maximum displacement does not occur at the minimum quality factor, but somewhere during the drop, where the observed quality factor has already been halved compared to the situation without any acoustic tube.

We can now propose an explanation for this effect in the light of our new model. When the two resonances are superimposed, the amplitude of the acoustic emission originating from the tuning fork motion becomes comparable with the resonant cavity eigenmode amplitude excited by the photoacoustic effect. Since those two acoustic sources are not in phase, an interference occurs and decreases the total pressure amplitude within the resonant cavity.

However, we stress that a reduced displacement does not necessarily mean that the resonant tuning fork is less suited for the QEPAS application. Indeed, the noise is also theoretically reduced when the quality factor of the whole system drops at the antiresonance (Eq. 15). Despite the fact that the signal (proportional to the displacement) drops, the signal to noise ratio does not display any antiresonance behavior, as shown in Fig. 8 (the analytical formula (15) is used in both cases for noise).

Actually, it may be more convenient to operate the resonant QEPAS technique at the antiresonance length of the tubes, because the signal to noise ratio is optimal while the quality factor and hence the response time is minimized. In Fig. 8, we can see that the numerical model (respectively,

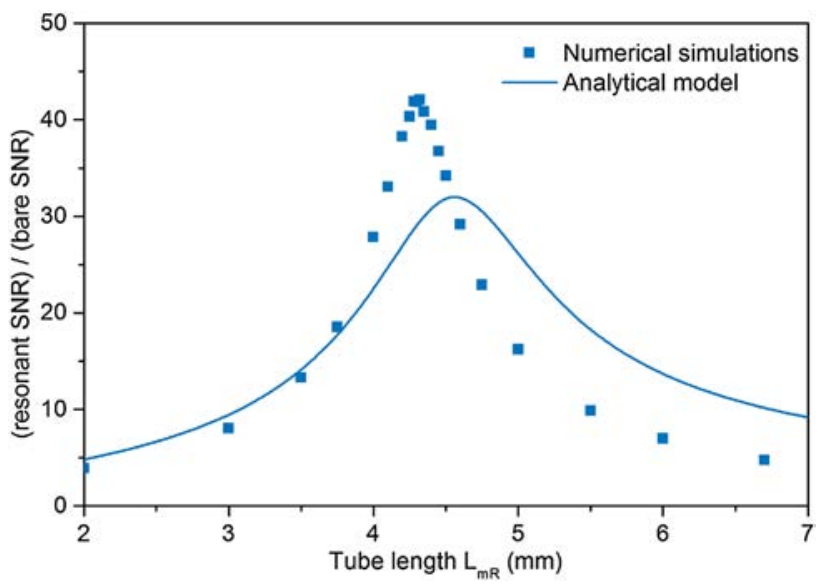

Fig. 8 Influence of the tube length $L_{m R}$ on the SNR gain provided by the addition of acoustic tubes. The displayed values are SNR values extracted from the resonant QEPAS modeling, normalized with the same SNR values that would be observed in the absence of tubes (bare tuning fork case). For the two models, we use equation (15) for the noise 
the analytical model) predicts an enhancement by a factor of 40 (resp. 30) with tubes of inner radius $R_{I}=0.46 \mathrm{~mm}$, in agreement with the optimal gain of 30 experimentally reported with slightly reduced inner radius of $R_{I}=0.3 \mathrm{~mm}$ [5]. We also remind that the height $h$ of the beam between the prongs is located $0.6 \mathrm{~mm}$ below the top opening in this paper. This parameter has, of course, an influence on the signal to noise ratio. In our analytical modelling, its dependency is hidden in the normalized overlap integral $O_{m}$, since the electric field distribution $u(r)$ depends on $h$ in a $3 \mathrm{D}$ space.

\subsection{Experiments using a commercial tuning fork}

To study the influence of the tube length on the system, we selected commercially available hypodermic needles with a radius of $R_{\mathrm{I}}=0.46 \mathrm{~mm}$ and outer diameter $R_{\mathrm{O}}=0.64 \mathrm{~mm}$. The needles have been chopped to various lengths ranging from 3 to $6 \mathrm{~mm}$.

The setup is identical to that described in the previous section; however, we chose a wavelength modulation technique instead of modulating the output power. A direct current of $I_{\mathrm{DC}}=300 \mathrm{~mA}$ is applied to the diode laser, while a small sinusoidal modulation (amplitude $I_{\mathrm{AC}}=15 \mathrm{~mA}$ ) is added at frequency $f_{0} / 2$ to modulate the wavelength across the central absorption peak of $\mathrm{CO}_{2}$ at $6490.05 \mathrm{~cm}^{-1}$. The measured average power entering the tubes is $P_{L}=23 \mathrm{~mW}$, and the power loss after the system is negligible.

An amplification circuit is used to enhance the signal originating from the tuning fork, and an SR530 lock-in amplifier is used to extract the component of the signal at frequency $f_{0}$. We choose a detection bandwidth of $\Delta f=0.063 \mathrm{~Hz}$, and we made sure that the Brownian motion of the tuning fork is the fundamental noise source of our system. The results are shown in Fig. 9.

In Fig. 9, the parameters of the analytical model $\left(C_{n m}, \omega_{m}\right.$, $Q_{a}$ and $O_{m}$ ) have been adjusted to obtain the best qualitative fit. Those parameters are of course the same for every tube lengths. The agreement is qualitatively good, and the model reproduces well the observed tendencies. Both the numerical and the analytical models are hence able to explain this experimental behavior, with the quality factor drop and the frequency shift when the two resonances are equal. The antiresonance is not clearly observed, even if it was predicted both analytically and numerically in the previous sections.

The optimal length of the tubes is found to be $L_{m R}=4.3$ $\mathrm{mm}$ (optimal SNR), even if the maximal signal is obtained for $L_{m R}=4.5 \mathrm{~mm}$ (optimal signal). This result is consistent with the value of $L_{m R}=4.4 \mathrm{~mm}$ reported in the literature [5]. At the optimum length, the quality factor is three times smaller than the one in the absence of tubes. The corresponding noise spectral density is measured at $250 \mu \mathrm{Vrms} . \mathrm{Hz}^{-1 / 2}$ in our setup. The signal to noise ratio is hence $\mathrm{SNR}=50$ $(1 \sigma)$, corresponding to the following normalized noise equivalent absorption (NNEA):

$\mathrm{NNEA}=\frac{510^{-6} \times 2310^{-3}}{50 \times 0.063}=9.210^{-9} \mathrm{~W} \mathrm{~cm}^{-1} \mathrm{~Hz}^{-1 / 2}$

This value is three times higher than the optimum NNEA values reported for a similar system of $3.310^{-9} \mathrm{~W} \mathrm{~cm}^{-1} \mathrm{~Hz}^{-1 / 2}$. The difference is due to the inner diameter of the tube, which is of $0.93 \mathrm{~mm}$ in our case whereas the optimal reported diameter is around $0.6 \mathrm{~mm}$.
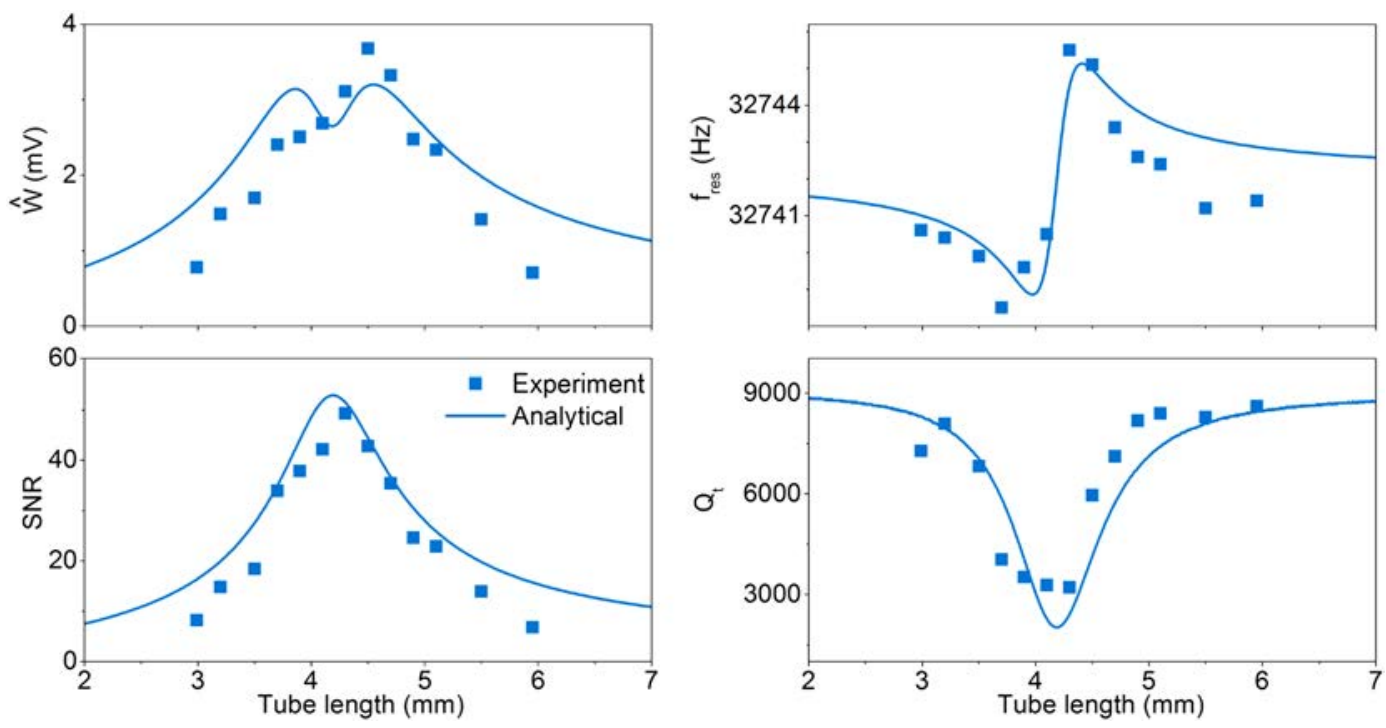

Fig. 9 Experimental influence of the tube length $L_{m R}$ on the tuning fork behavior. The parameters of the analytical model $\left(C_{n m}, \omega_{m}, Q_{a}\right.$ and $\left.O_{m}\right)$ have been adjusted to obtain the best qualitative fit with the experimental data 


\section{Conclusion}

In this article, we have derived a new analytical model to describe the behavior of a resonant QEPAS setup. Taking into account the acoustic emission of the mechanical resonator, we have obtained a generalized fundamental equation for photoacoustic able to describe the possible coupling with an acoustic resonance. The model reproduces accurately every tendencies obtained with numerical simulations using a finite element commercial software, including the change in the resonance frequency, the antiresonance behavior and the drop of the quality factor. The model is then used to fit experimental data, which confirmed the pertinence of our approach. The model can be adapted to any configuration, since an expression for the acoustic eigenmode can always be obtained using finite element simulations. We also believe that the acoustic emission of the tuning fork together with the resonant nature of the acoustic field could be responsible for the discrepancy previously reported for the bare QEPAS theory [11]. The experiments were twice more sensitive than the theoretical result, which could be explained by a weak acoustic resonance appearing between the two prongs and enhancing the pressure forces. Similar to our new analytical description of resonant QEPAS, the assumption of an acoustic field unaffected by the presence of the tuning fork must now be considered with caution. The use of resonant acoustic description can now be used to better understand resonant QEPAS systems, towards quantitative optimization of resonant QEPAS designs.

\section{References}

1. C.K.N. Patel, Laser photoacoustic spectroscopy helps fight terrorism: high sensitivity detection of chemical Warfare Agent and explosives. Eur. Phys. J. Special Topics 153(1), 1-18 (2008)

2. S.M. Cristescu, S.T. Persijn, S. TE Lintel Hekkert, F.J.M. Harren, Laser-based systems for trace gas detection in life sciences. Appl. Phys. B 92(3), 343-349 (2008)

3. A. Miklós, P. Hess, Z. Bozóki, Application of acoustic resonators in photoacoustic trace gas analysis and metrology. Rev. Sci. Instrum. 72(4), 1937 (2001)

4. A.A. Kosterev, F.K. Tittel, Chemical sensors based on quantum cascade lasers. IEEE J. Quantum Electron. 38(6), 582-591 (2002)

5. L. Dong, A.A. Kosterev, D.M. Thomazy, F.K. Tittel, QEPAS spectrophones: design, optimization, and performance. Appl. Phys. B 100(3), 627-635 (2010)

6. H. Yi, K. Liu, S. Sun, W. Zhang, Xiaoming Gao, Theoretical analysis of off beam quartz-enhanced photoacoustic spectroscopy sensor. Optics Commun. 285(24), 5306-5312 (2012)

7. H. Yi, W. Chen, A. Vicet, Z. Cao, T-shape microresonator-based quartz enchanced photoacoustic spectroscopy for ambient methane monitoring using $3.38 \mu \mathrm{m}$ antimonide-distributed feedback laser diode. Appl. Phys. B 116, 423-428 (2014)

8. P. Patimisco, G. Scamarcio, F.K. Tittel, V. Spagnolo, Quartzenhanced photoacoustic spectroscopy: a review. Sensors 14, 61656206 (2014)
9. A.A. Kosterev, J.H. Doty, Resonant optothermoacoustic detection: technique for measuring weak optical absorption by gases and micro-objects. Optics Lett. 35(21), 3571-3573 (2010)

10. N. Petra, J. Zweck, S.E. Minkoff, A.A. Kosterev, J.H. Doty III, Modeling and design optimization of a resonant optothermoacoustic trace gas sensor. SIAM J. Appl. Math. 71(1), 309-332 (2011)

11. N. Petra, J. Zweck, A.A. Kosterev, S.E. Minkoff, D. Thomazy, Theoretical analysis of a quartz-enhanced photoacoustic spectroscopy sensor. Appl. Phys. B 94(4), 673-680 (2009)

12. D.V. Serebryakov, I.V. Morozov, A.A. Kosterev, V.S. Letokhov, Laser microphotoacoustic sensor of ammonia traces in the atmosphere. Quantum Electron. 40(2), 167-172 (2010)

13. H. Yi, W. Chen, S. Sun, K. Liu, T. Tan, T-shape microresonatorbased high sensitivity quartz-enhanced photoacoustic spectroscopy sensor. Optics Express 20(8), 9187-9196 (2012)

14. S.L. Firebaugh, F. Roignant, E.A. Terray. Modeling the response of photoacoustic gas sensors. In COMSOL, number 1 (2009)

15. S.L. Firebaugh, E.A. Terray, L. Dong. Optimization of resonator radial dimensions for quartz enhanced photoacoustic spectroscopy systems. Proc. SPIE 8600, 86001S-1-86001S-12 (2013)

16. Yingchun Cao, Wei Jin, Hoi Lut Ho, Optimization of spectrophone performance for quartz-enhanced photoacoustic spectroscopy. Sensors Actuators B 174, 24-30 (2012)

17. U. Willer, M. Köhring, M. Mordmüller, W. Schade, Photoacoustic sensing with micro-tuning forks. Proceedings of SPIE 9482B, 1-11 (2015)

18. G. Aoust, R. Levy, B. Bourgeteau, O. Le Traon, Viscous damping on flexural mechanical resonators. Sensors Actuators A Phys. 230, 126-135 (2015)

19. L. Meirovitch. Elements of Vibration Analysis (McGraw-Hill, Singapore, 1986). ISBN 0-07-041342-8

20. G. Aoust, R. Levy, B. Bourgeteau, O. Le Traon, Acoustic damping on flexural mechanical resonators. Sensors Actuators A Phys. 238, 158-166 (2016)

21. M.P. Morse, H. Feshbach, Methods of Theoretical Physics (Mc Graw-Hill Book Compagny Inc, New York, 1953)

22. R.M. Sillitto, Angular distribution of the acoustic radiation from a tuning fork. Am. J. Phys. 34(8), 639-644 (1966)

23. A.A. Kosterev, F.K. Tittel, D.V. Serebryakov, A.L. Malinovsky, I.V. Morozov, Applications of quartz tuning forks in spectroscopic gas sensing. Rev. Sci. Instrum. 76(4), 043105 (2005)

24. G. Wysocki, A.A. Kosterev, F.K. Tittel, Influence of molecular relaxation dynamics on quartz-enhanced photoacoustic detection of $\mathrm{CO} 2$ at $\lambda=2$ microns. Appl. Phys. B 85, 301-306 (2006)

25. L.S. Rothman, I.E. Gordon, A. Barbe, D.C. Benner, P.F. Bernath, M. Birk, V. Boudon, L.R. Brown, A. Campargue, J. Champion, K. Chance, L.H. Coudert, V. Dana, V.M. Devi, S. Fally, J. Flaud, R.R. Gamache, A. Goldman, D. Jacquemart, I. Kleiner, N. Lacome, W.J. Lafferty, J. Mandin, S.T. Massie, S.N. Mikhailenko, C.E. Miller, N. Moazzen-ahmadi, O.V. Naumenko, A.V. Nikitin, J. Orphal, V.I. Perevalov, A. Perrin, A. Predoi-cross, C.P. Rinsland, M. Rotger, S.A. Tashkun, J. Tennyson, R.A. Toth, A.C. Vandaele, J. Vander Auwera, The HITRAN, molecular spectroscopic database. J. Quant. Spectrosc. Radiat. Transfer 110(533-572), 2009 (2008)

26. S. Paquay. Développement $d$ ' une méthodologie de simulation numérique pour les problèmes vibro-acoustiques couplés intérieurs / extérieurs de grande taille. 2001

27. s. a. Open Engineering. OOFELIE::Multiphysics (2013)

28. M.J. Moloney, D.L. Hatten, Acoustic quality factor and energy losses in cylindrical pipes. Am. J. Phys. 69(3), 311 (2001)

29. L.D. Landau, E.M. Lifshitz. Fluid Mechanics (Pergamon Press, Oxford, 1959) 